\title{
Small Size Dual-band Bandpass Filters with Multiconductor Transmission Lines and Shunt Open Stubs
}

\author{
Mario Pérez-Escribano ${ }^{\# 1}$, Juan José Sánchez-Martínez ${ }^{\# 2}$ and Enrique Márquez-Segura ${ }^{\# 3}$ \\ Departamento de Ingeniería de Comunicaciones, E.T.S. Ingeniería de Telecomunicación, Universidad de Málaga \\ Campus de Teatinos s/n, E-29071 Málaga (Spain) \\ $\left\{{ }^{1} \mathrm{mpe},{ }^{2} \mathrm{jjsm},{ }^{3} \mathrm{ems}\right\} @$ ic.uma.es
}

\begin{abstract}
A dual-band bandpass filter consisting of multiconductor transmission lines (MTL) and shunt stubs has been designed. The used topology, based on the interconnection of two identical MTL and a shunt open stub, has a frequency response that can be modelled by using the generalized Chebyshev functions. A prototype of a 4 fingers-MTL is manufactured and measured and a good agreement between analytical and measured results is obtained. Furthermore, it is easy to get a design criterion that enables getting good responses varying just a few parameters.
\end{abstract}

Keywords - Dual-band bandpass filter, Multiconductor transmission lines, shunt stubs.

\section{INTRODUCTION}

The use of double bands and broader bandwidths is essential in new communication systems. For this reason, the design of dual band filters has been addressed by different authors in the last years. Although the first band has been controlled from the beginning of the filters synthesis, the control of the second band was not addressed until a few years ago [1]. Some of the authors have based their works on finding techniques for miniaturizing or making compact filters [2], [3], others in finding controllable bands [4], [5] or exploring new fabrication technologies [6], [7].

Coupled lines has been used traditionally in the design of filters, because of the easiness to get good responses varying few parameters. Nevertheless, using more than two coupled lines can offer more variety in the kind of responses that can be achieved. For example, multiconductor transmission lines (MTL) have been used for synthesizing wide and ultra-wideband filters [8]-[10].

The capability of using MTLs for creating dual-band responses is discussed here. A novel prototype, composed of two short-circuited identical MTLs and a shunt open stub is presented and assessed. By changing the line-width and line-spacing of the MTL and the width of the stub, the main parameters of the filter can be controlled. Moreover, the electrical length of both $(\theta)$ will determine the the middle frequency $\left(f_{0}\right)$ of the desired two bands. Furthermore, by using planar MTLs it is possible to obtain frequency responses that would be unapproachable with a traditional pair of coupled lines. The circuital model of the filter has been developed, including a design criterion for the central frequency of each band and its bandwidth. Finally, an experimental validation has been performed and the results of the analytical model and the measurements are compared.

\section{DESIGN PROCEDURE}

The circuit analyzed in this work for dual-band BPFs is shown in Fig. 1. The analysis of these structures follows a similar procedure to the described in [11]-[13]. The synthesis procedure consists of computing the frequency responses of the circuit (i.e., the $S_{21}$ parameter), obtaining a proper filtering function for synthesizing dual-band BPFs and finally, determining the circuit design parameters.

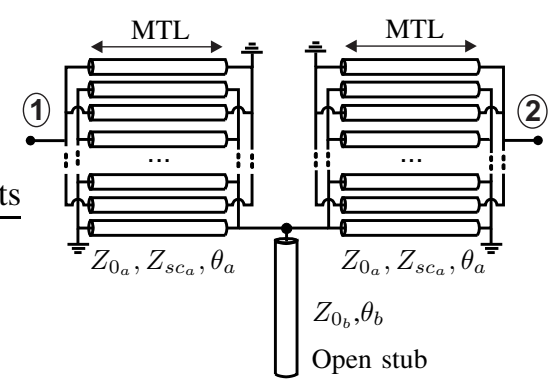

Fig. 1. Transmission line equivalent circuit model for the analyzed dual-band bandpass filter.

The proposed circuit (see Fig. 1) is composed of two series short-circuited MTL and a shunt open stub. This prototype is considered in order to design first-order dual-band BPFs. However, this order could be increased by cascading several filter sections. The analysis of the filter can be straightforwardly accomplished by using the equivalent circuit for a short-circuited MTL [14], consisting of a transmission-line section and two shunt short-circuited stubs with characteristic impedances $Z_{0_{a}}$ and $Z_{s c_{a}}$, respectively. Fig. 2 depicts the equivalent circuit transmission-line model for the proposed prototype, where $Z_{0_{a}}$ and $Z_{s c_{a}}$ are given by

$$
\begin{array}{r}
Z_{0_{a}}=\frac{2 Z_{o o}}{(k-1)\left(1-\frac{Z_{o o}}{Z_{o e}}\right)} \\
Z_{s c_{a}}=\frac{Z_{o e}+Z_{o o}}{1+\frac{Z_{o o}}{Z_{o e}}(k-1)}
\end{array}
$$




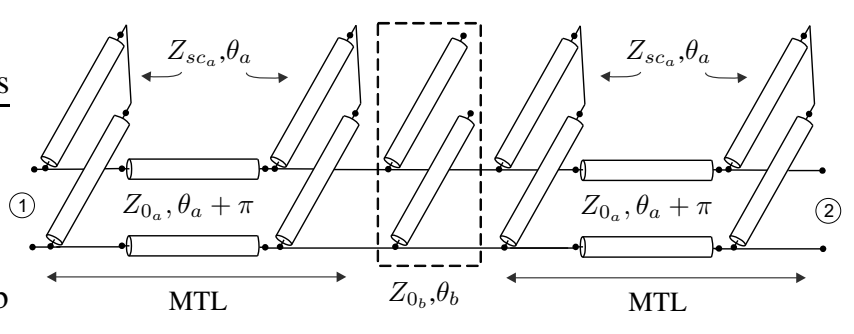

Fig. 2. Equivalent circuit model for the proposed filters.

$k$ denotes the number of conductors and $Z_{o e}$ and $Z_{o o}$ are the even- and odd-mode impedances of a pair of coupled lines, whereas $\theta_{a}$ is the electrical length of a MTL, computed as the arithmetic mean value of the even- and odd-mode electrical lengths.

The open-circuited stubs are defined by the characteristic impedance $Z_{0_{b}}$ and the electrical length $\theta_{b}$. To analyse these circuits the even- and odd-mode analysis is employed. The even- $\left(Z_{i n_{e}}\right)$ and odd-mode $\left(Z_{i n_{o}}\right)$ input impedances are given by

$$
\begin{gathered}
Z_{i n_{e}=j Z_{0_{a}} Z_{s c_{a}}} \tan (\theta) \frac{\left(Z_{0_{a}} Z_{s c_{a}}-2 Z_{0_{b}}\left(Z_{0_{a}}+Z_{s c_{a}}\right) \cot ^{2}(\theta)\right)}{\Delta_{1}} \\
Z_{i n_{o}}=j \frac{Z_{0_{a}} Z_{s c_{a}} \tan (\theta)}{Z_{0_{a}}+Z_{s c_{a}}} \\
\Delta_{1}=Z_{0_{a}}^{2} Z_{s c_{a}}+Z_{0_{a}} Z_{s c_{a}}^{2}+2 Z_{0_{b}} Z_{s c_{a}}^{2} \\
-2 \cot ^{2}(\theta) Z_{0_{b}}\left(Z_{0_{a}}^{2}+2 Z_{0_{a}} Z_{s c_{a}}\right)
\end{gathered}
$$

and the S-parameters of the filters are determined as

$$
\begin{array}{r}
S_{11}=\frac{Z_{i n_{e}} Z_{i n_{o}}-Z_{0}^{2}}{\Delta_{2}}, \quad S_{21}=\frac{Z_{0}\left(Z_{i n_{e}}-Z_{i n_{o}}\right)}{\Delta_{2}} \\
\Delta_{2}=Z_{0}^{2}+Z_{0}\left(Z_{i n_{e}}+Z_{i n_{o}}\right)+Z_{i n_{e}} Z_{i n_{o}}
\end{array}
$$

being $Z_{0}$ the characteristic impedance used to terminate the input and output ports. These expressions can be simplified if the wire-bonded MTL and the open stubs are chosen to be a quarter-wavelength long at the middle frequency $\left(f_{o}\right)$ of the desired two passbands. Besides, assuming a TEM propagation, it can be considered that

$$
\theta_{a}=\theta_{b}=\theta=\theta_{o} \frac{f}{f_{o}}=\frac{\pi}{2} \frac{f}{f_{o}} .
$$

The feasibility will depend on the physical realizability of the required values of $Z_{0_{a}}, Z_{s c_{a}}$ and $Z_{0_{b}}$, which conditions the operating bandwidths and the pass band frequency ratio. Fig. 3 represents the achievable values of $Z_{0_{a}}$ and $Z_{s c_{a}}$ as a function of the line width $W$ and spacing $S$ for different numbers of conductors $k$. These curves have been calculated using the Rogers 4350B substrate, with a dielectric constant of 3.66, a loss tangent of 0.0031 and thickness of 30 mils. This substrate will be use for the prototype developed in this work. It is straightforward to observe that by increasing the number of conductors makes possible to reduce the values of $Z_{0_{a}}$, that can be directly translated into wider bandwidths and

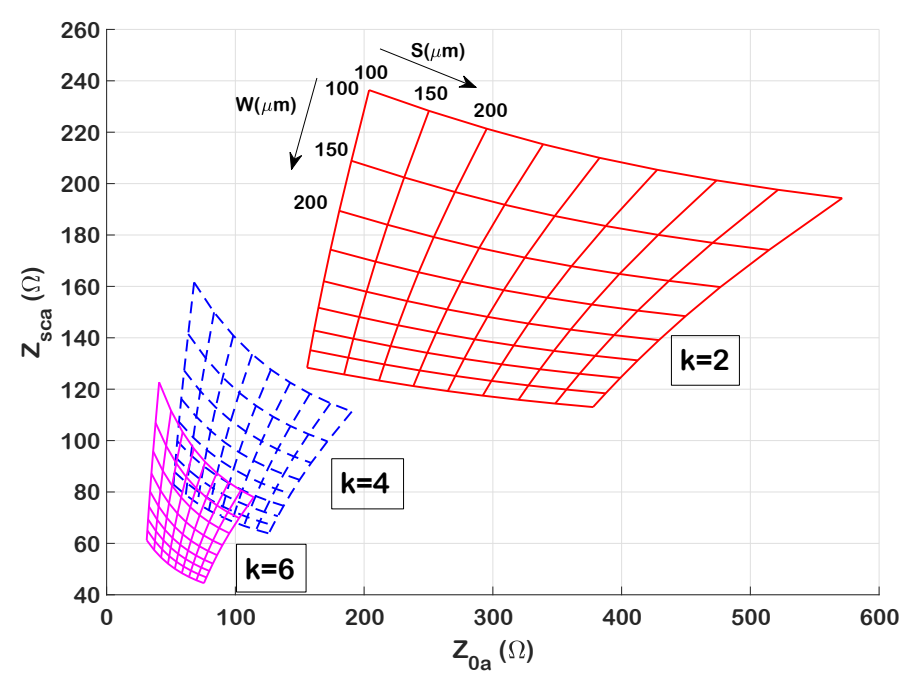

Fig. 3. Analytical values of impedances $Z_{0_{a}}$ and $Z_{s c_{a}}$ depending on the number of conductors $(k)$, their width $(W)$ and the separation between them $(S)$.

higher frequency pass band ratios. Therefore, in this point is important to highlight the advantages of using multiconductor transmission lines instead of only a pair of coupled lines.

From Eq. 3 and after some algebraic transformations, including the condition

$$
Z_{0}=\frac{Z_{0_{a}} Z_{s c_{a}}}{Z_{0_{a}}+Z_{s c_{a}}}
$$

that must be satisfied by the designed filter and that allows to synthesize a generalized four order Chebyshev function [15], the squared magnitude of $S_{11}$ and $S_{21}$ can be expressed as

$$
\begin{aligned}
& \left|S_{11}\right|^{2}=\frac{F_{I}^{2}}{1+F_{I}^{2}}, \quad\left|S_{21}\right|^{2}=\frac{1}{1+F_{I}^{2}} \\
& F_{I}=\frac{g_{4} \tan ^{4}(\theta)+g_{2} \tan ^{2}(\theta)+g_{0}}{\tan (\theta)\left(1+\tan ^{2}(\theta)\right)}, K_{I}=\frac{\bar{Z}_{0_{a}}^{2}}{2 \bar{Z}_{0_{b}}} \\
& g_{4}=K_{I}, \quad g_{2}=\left(1+\frac{2 \bar{Z}_{0_{b}}}{\bar{Z}_{0_{a}}^{2}}\left(1-\bar{Z}_{0_{a}}^{2}\right)\right) K_{I} \\
& g_{0}=-2 \bar{Z}_{0_{b}}\left(1-\frac{1}{\bar{Z}_{0_{a}}^{2}}\right) K_{I}
\end{aligned}
$$

where

$$
\bar{Z}_{0_{a}}=Z_{0_{a}} / Z_{0}, \quad \bar{Z}_{0_{b}}=Z_{0_{b}} / Z_{0}
$$

The frequency response of the structure is perfectly characterized by means of (6), and taking into account the form of $F_{I}$, it is possible to design dual-band bandpass filters with real transmission zeros at $\theta_{z}=z \frac{\pi}{2}, z=0,1,2, \ldots$ These transmission zeros are used to enhance both, the isolation between two pass-bands and the rejection level in the lower and upper stop-bands. Being a structure adjusted to quarter-wavelenght long, there will be replicas of the bands at 


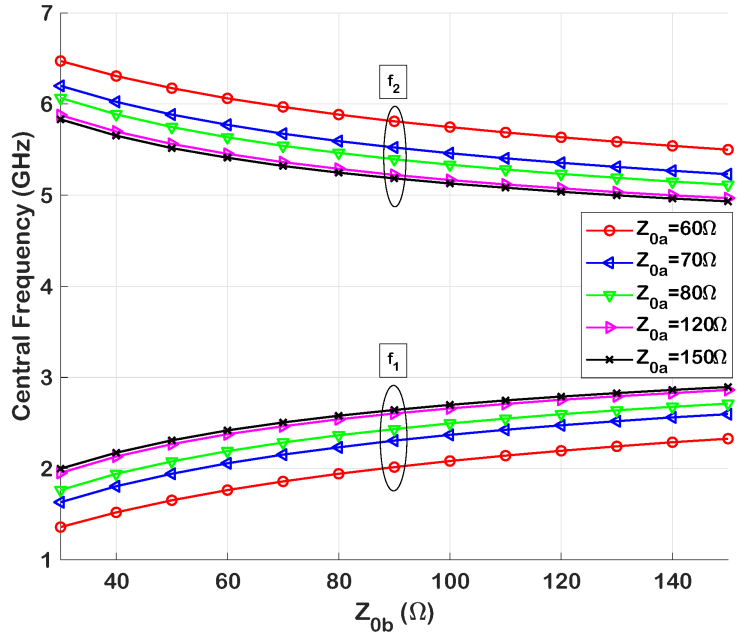

Fig. 4. Analytical values of the central frequency of both bands for different impedances $Z_{0_{a}}$ and $Z_{0_{b}}$ combinations and $f_{0}=3.90 \mathrm{GHz}$.

higher frequencies. The proposed structure could be viewed as a wideband filter split into two by the stubs [16].

Figs. 4 and 5 show the analytical values of the central frequency of each band ( $f_{1}$ and $\left.f_{2}\right)$ and the bandwidth (using this topology, both bands have the same bandwidth, being impossible to control it separately in each band) for several values of $Z_{0_{a}}$ and $Z_{0_{b}}$. As seen in Fig. 4, central frequencies approach each other when increasing both $Z_{0_{a}}$ and $Z_{0_{b}}$. On the other hand, Fig. 5 shows that the bandwidth increases with the value of $Z_{0_{b}}$, but decreases with $Z_{0_{a}}$. It is important to mention that when $Z_{0_{a}}$ is close to $Z_{0}$, the range of possible values of the bandwidth and the central frequencies are much larger than the one when the value of $Z_{0_{\alpha}}$ is higher. Nevertheless, an important condition that must be taken into account, obtained from Eq. 5, is that $Z_{0_{a}}>Z_{0}$ and $Z_{s c_{a}}>Z_{0}$, because it is necessary a positive value of $Z_{0_{a}}$ and $Z_{s c_{a}}$ to achieve a double band bandpass response. For this reason, not all the possible combinations of $W, S$ and $k$ are valid for designing this kind of filters, so it is the main limitation when designing.

\section{EXPERIMENTAL VALIDATION}

\section{A. Design}

As a design example, a prototype has been manufactured in the Rogers 4350B substrate. It has been used 4-finger MTL, whose width is $w_{f}=180 \mu \mathrm{m}$, separated by $s_{f}=214 \mu \mathrm{m}$. For the stub, a line of width $w_{s}=685 \mu \mathrm{m}$ has been used. If the parameters of the MTLs and the stub plus a dispersion model for microstrip and coupled microstrip lines are considered, the values for the characteristic impedances are $Z_{0_{a}}=90 \Omega$, $Z_{s c_{a}}=112.5 \Omega$ and $Z_{0_{b}}=80 \Omega$, using a reference impedance $Z_{0}=50 \Omega$.

The filter has been designed for dual band WiFi applications, choosing the central frequencies of the bands at $f_{1}=2.42 \mathrm{GHz}$ and $f_{2}=5.38 \mathrm{GHz}$. Therefore, the value for the middle frequency $\left(f_{0}\right)$ is $3.90 \mathrm{GHz}$. For this frequency, and once dispersion has been taken into account, the lengths of the

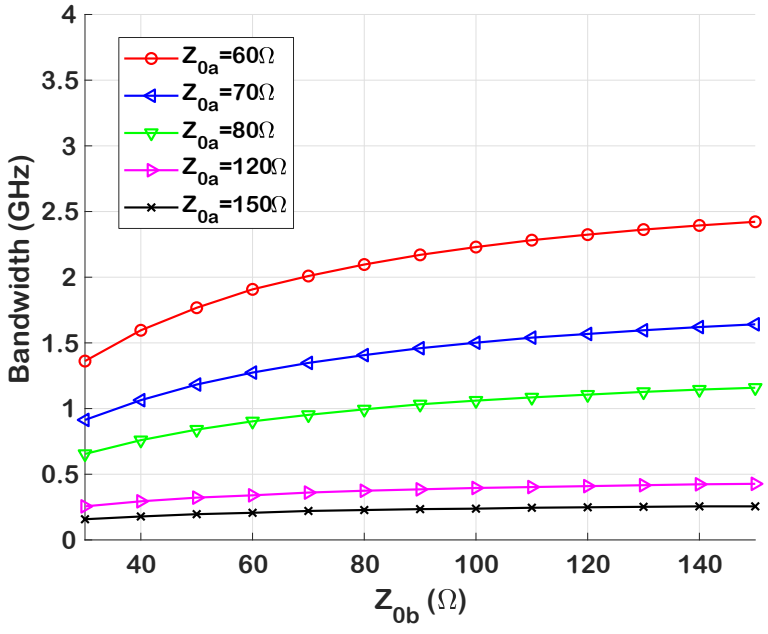

Fig. 5. Analytical values of the filter bandwidth for different impedances $Z_{0_{a}}$ and $Z_{0_{b}}$ combinations and $f_{0}=3.90 \mathrm{GHz}$.

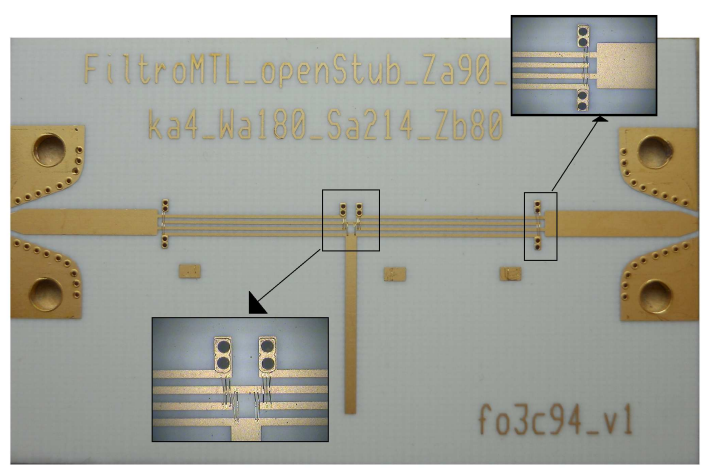

Fig. 6. Fabricated prototype with 4-finger MTLs and a shunt open stub

MTLs and the stub are $12 \mathrm{~mm}$. A photograph of the fabricated prototype is shown in Fig. 6.

\section{B. Results}

Theoretical S-parameters are calculated by considering the equations for a k-line quarter-wavelength four-port coupler [17] and the Kirschning and Jansen dispersion model [18]. Once these parameters have been calculated, ABCD-parameters of both MTL and stub are computed and a cascade connection is performed. The results are transformed into S-parameters. Furthermore, it is important to highlight that design parameters are computed by means of the expressions in section II, without any optimization process made by an electronic simulator. Measurements have been done using Agilent PNA-X 5257A, after a TRL calibration.

Analytical and measured $S_{11}$ and $S_{21}$ and the group delay are represented in Fig. 7. As designed, the first band has a central frequency $f_{1}=2.43 \mathrm{GHz}$, whereas the second band has a central frequency $f_{2}=5.39 \mathrm{GHz}$. As it can be seen, there is an excellent agreement between analytical and measured curves in Fig. 7. The difference between analytical and measured $f_{0}$ is due to the effective length of the stub and 

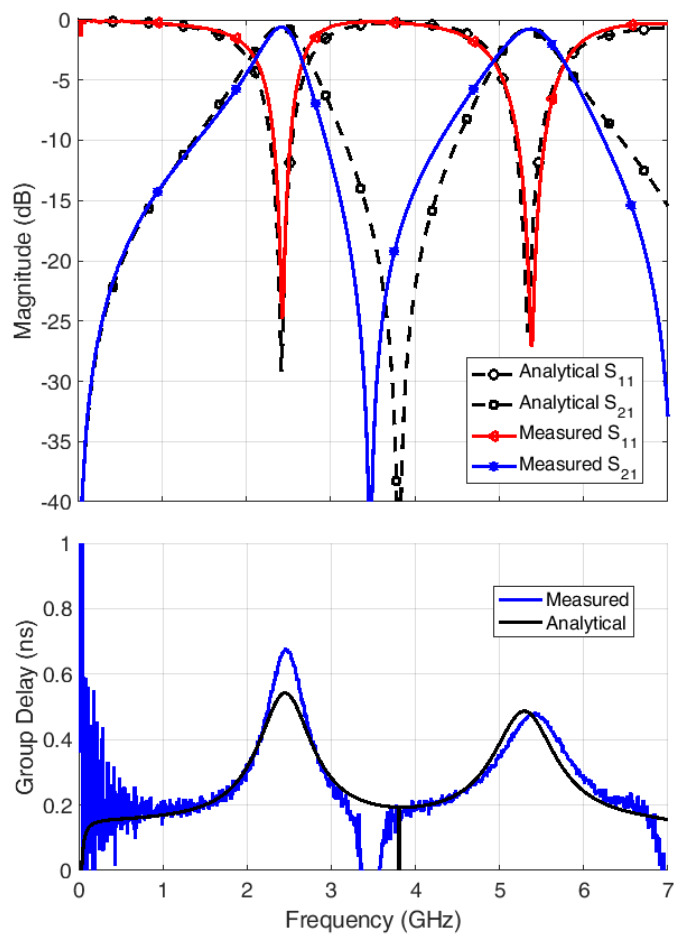

Fig. 7. Analytical and measured scattering matrix elements $\left(S_{11}\right.$ and $\left.S_{21}\right)$ of the manufactured prototype.

the length increase of the MTLs because of the interconnection with the stub.

\section{CONCLUSION}

The capability of using multiconductor transmission lines in order to synthesize dual band pass filters has been analysed and discussed in this article. Two identical short-circuited multiconductor transmission lines and a shunt open stub configuration have been proposed and closed-form design equations have been given. This structure has a dual-band bandpass response, that allows to control the central frequency of the filter varying just the lengths of the MTLs and the line. Furthermore, the central frequency of each band and its bandwidth can be easily controlled by choosing different combinations of the values of the impedances of the MTLs and the open stub. In addition, an excellent agreement between analytical and measured results has been assessed without the need for any optimization process using a full-wave electromagnetic simulation.

\section{ACKNOWLEDGMENT}

This work has been supported by the Spanish Ministerio de Economía, Industria y Competitividad under Project ADDMATE TEC2016-76070-C3-3-R (AEI/FEDER, UE) and by the Spanish Ministerio de Educación, Cultura y Deporte under Grant FPU16/00246.

\section{REFERENCES}

[1] C.-M. Tsai, H.-M. Lee, and C.-C. Tsai, "Planar filter design with fully controllable second passband," IEEE Trans. Microw. Theory Techn., vol. 53, no. 11, pp. 3429-3439, Nov 2005.

[2] H. T. Ziboon and J. K. Ali, "Compact dual-band bandpass filter based on fractal stub-loaded resonator," in 2017 Progress In Electromagnetics Research Symposium - Spring (PIERS), May 2017, pp. 1815-1819.

[3] J. Xu, Z. Y. Chen, and Q. H. Cai, "Design of miniaturized dual-band low-pass-bandpass and bandpass filters," IEEE Trans. Compon. Packag. Manuf. Technol., vol. 8, no. 1, pp. 132-139, Jan 2018.

[4] J. X. Chen, M. Z. Du, Y. L. Li, Y. J. Yang, and J. Shi, "Independently tunable/controllable differential dual-band bandpass filters using element-loaded stepped-impedance resonators," IEEE Trans. Compon. Packag. Manuf. Technol., vol. 8, no. 1, pp. 113-120, Jan 2018.

[5] I. Wangshuxing, D. Zhou, D. Zhang, and S. Han, "Dual-band bandpass filter using loop resonator with independently-tunable passband," Electronics Letters, vol. 53, no. 25, pp. 1655-1657, 2017.

[6] C.-W. Tang, S.-F. You, and I.-C. Liu, "Design of a dual-band bandpass filter with low-temperature co-fired ceramic technology," IEEE Trans. Microw. Theory Techn., vol. 54, no. 8, pp. 3327-3332, Aug 2006.

[7] Y. Takagi, K. Satoh, and S. Narahashi, "New approach for configuring a parallel-planar dual-band bandpass filter by employing multilayered ltcc technologies," in 2012 IEEE Radio and Wireless Symposium, Jan 2012, pp. $147-150$

[8] J. J. Sánchez-Martínez, E. Márquez-Segura, and S. Lucyszyn, "Design of Compact Wideband Bandpass Filters Based on Multiconductor Transmission Lines With Interconnected Alternate Lines," IEEE Microw. Wireless Compon. Lett., vol. 24, no. 7, pp. 454-456, Jul. 2014.

[9] J. J. Sánchez-Martinez, E. Márquez-Segura, and S. Lucyszyn, "Synthesis and design of high-selectivity wideband quasi-elliptic bandpass filters using multiconductor transmission lines," IEEE Trans. Microw. Theory Techn., vol. 63, no. 1, pp. 198-208, Jan 2015.

[10] J. J. Sánchez-Martínez and E. Márquez-Segura, "Analytical design of wire-bonded multiconductor transmission-line-based ultra-wideband differential bandpass filters," IEEE Trans. Microw. Theory Techn., vol. 62, no. 10, pp. 2308-2315, Oct 2014.

[11] R. Zhang and L. Zhu, "Synthesis and Design of Wideband Dual-Band Bandpass Filters With Controllable In-Band Ripple Factor and Dual-Band Isolation," IEEE Trans. Microw. Theory Techn., vol. 61, no. 5, pp. 1820-1828, May 2013.

[12] R. Z. add L. Zhu, "Synthesis of dual-wideband bandpass filters with source-load coupling network," IEEE Trans. Microw. Theory Techn., vol. 62, no. 3, pp. 441-449, March 2014.

[13] I. C. Hunter, Theory and Design of Microwave Filters, Stevenage, Ed. U.K.: IEE Press, 2001.

[14] J. J. Sánchez-Martínez and E. Márquez-Segura, "Analysis of wire-bonded multiconductor transmission line-based phase-shifting sections," J. Electromagnet. Wave., vol. 27, no. 16, pp. 1997-2009, Sep. 2013.

[15] S. Amari, F. Seyfert, and M. Bekheit, "Theory of Coupled Resonator Microwave Bandpass Filters of Arbitrary Bandwidth," IEEE Trans. Microw. Theory Techn., vol. 58, no. 8, pp. 2188-2203, Aug 2010.

[16] L.-C. Tsai and C.-W. Hsue, "Dual-band bandpass filters using equal-length coupled-serial-shunted lines and z-transform technique," IEEE Trans. Microw. Theory Techn., vol. 52, no. 4, pp. 1111-1117, April 2004.

[17] W. Ou, "Design Equations for an Interdigitated Directional Coupler," IEEE Trans. Microw. Theory Techn., vol. 23, no. 2, pp. 253-255, Feb. 1975.

[18] M. Kirschning and R. Jansen, "Accurate Wide-Range Design Equations for the Frequency-Dependent Characteristic of Parallel Coupled Microstrip Lines," IEEE Trans. Microw. Theory Techn., vol. 32, no. 1, pp. 83-90, Jan. 1984. 